\title{
The Extended Symmetry Lie Algebra and the Asymptotic Expansion of the Transversal Correlation Function for the Isotropic Turbulence
}

\author{
V. N. Grebenev, ${ }^{1}$ A. N. Grishkov, ${ }^{2}$ and M. Oberlack ${ }^{3}$ \\ ${ }^{1}$ Institute of Computational Technologies, Russian Academy of Science, Lavrentjev Avenue 6, \\ Novosibirsk 630090, Russia \\ ${ }^{2}$ Institute of Mathematics and Statistics, University of Sao Paulo, Rua do Matao 1010, 66281 Sao Paulo, SP, Brazil \\ ${ }^{3}$ Technical University Darmstadt, Department of Mechanical Engineering, Petersenstrasse 30, \\ 64287 Darmstadt, Germany \\ Correspondence should be addressed to V. N. Grebenev; vngrebenev@gmail.com
}

Received 4 January 2013; Accepted 12 February 2013

Academic Editor: Rutwig Campoamor-Stursberg

Copyright (c) 2013 V. N. Grebenev et al. This is an open access article distributed under the Creative Commons Attribution License, which permits unrestricted use, distribution, and reproduction in any medium, provided the original work is properly cited.

The extended symmetry of the functional of length determined in an affine space $K^{3}$ of the correlation vectors for homogeneous isotropic turbulence is studied. The two-point velocity-correlation tensor field (parametrized by the time variable $t$ ) of the velocity fluctuations is used to equip this space by a family of the pseudo-Riemannian metrics $\mathrm{dl}^{2}(t)$ (Grebenev and Oberlack (2011)). First, we observe the results obtained by Grebenev and Oberlack (2011) and Grebenev et al. (2012) about a geometry of the correlation space $K^{3}$ and expose the Lie algebra associated with the equivalence transformation of the above-mentioned functional for the quadratic form $d l_{D^{2}}^{2}(t)$ generated by $d l^{2}(t)$ which is similar to the Lie algebra constructed by Grebenev et al. (2012). Then, using the properties of this Lie algebra, we show that there exists a nontrivial central extension wherein the central charge is defined by the same bilinear skew-symmetric form $c$ as for the Witt algebra which measures the number of internal degrees of freedom of the system. For the applications in turbulence, as the main result, we establish the asymptotic expansion of the transversal correlation function for large correlation distances in the frame of $d l_{D^{2}}^{2}(t)$.

\section{Introduction}

This paper is a continuation of [1-3] wherein we investigated both the geometry and the group of transformations of an affine space $K^{3}$ of the correlation vectors. In [1], we used the two-point velocity-correlation tensor to equip the correlation space $K^{3}$ by the structure of a pseudo-Riemannian manifold of a variable signature and gave the geometric realization of the two-point velocity-correlation tensor which presents a metric tensor in the case of homogeneous isotropic turbulence. This construction presents the template for embedding the couple $\left(K^{3}, d l^{2}(t)\right)$ into the Euclidean space $\mathbb{R}^{3}$ with the standard metric. The Lagrangian system in the extended space $K^{3} \times R_{+}$was introduced in [3] that allowed us to attract common concept and technics of the Lagrangian mechanics for the application in turbulence. Dynamics in time of a singled out fluid volume equipped with a family of pseudo-Riemannian metrics was described in the frame of the geometry generated by $d l^{2}(t)$ whose components are the correlation functions that evolve according to the von Kármán-Howarth equation [4]. Notice here that the first integrals of the equations of geodesic curves form the "kinematic" conservation laws; see, for more details, [3]. In [2], we considered the functional of length and studied the infinitesimal transformations admitted by this functional. We extended the variational symmetries up to the equivalence transformations which generate an infinite-dimensional Lie algebra (the so-called extended symmetries algebra). The 
properties obtained of this algebra were discussed both for the signature $(++)$ and for the signature $(+-)$ of the abovementioned pseudo-Riemannian metrics.

The main result of the paper is the derivation of the asymptotic expansion of the transversal correlation function for large correlation distances. Notice that this expansion is obtained in the case when the correlation space $K^{3}$ is equipped with the metric generated by the twopoint velocity-correlation tensor. This derivation is based on the algebraic constructions as in Conformal Field Theory (CFT). Also we present a nontrivial extension of the abovementioned infinite-dimensional Lie algebra.

Central extensions of groups and Lie algebras occur in CFT in the investigations of conformal symmetries where the Virasoro algebra is used as the fundamental set of infinitesimal symmetries. This implies the existence of an infinite number of independent constraints, which yields the exceptional feature of the two-dimensional conformal theory. This algebra is the Lie algebra of the central extension of the group of diffeomorphisms of the circle whose basic elements $\left\{L_{n}\right\}, n \in Z$, satisfy the following commutation relations:

$$
\left[L_{n}, L_{m}\right]=(n-m) L_{n+m}+\frac{c}{12} n\left(n^{2}-1\right) \delta_{n+m}
$$

where $\delta_{k}=1$ for $k=0$ and $\delta_{k}=0$ for $k \neq 0$. The quantity $c$ is known as the central charge, and its value in general depends on the particular theory under consideration. For the application in turbulence in $[5,6]$, the central charge $c$ is expressed via the diffusion coefficient $\kappa$ of SLE: the ShramLövner evolution, of random curves in planar domains; see $[5,6]$. Here, the diffusion coefficient $\kappa$ allows us to classify the conformally invariant random curves into classes denoted by $\mathrm{SLE}_{\kappa}$. The formula $c=(8-3 \kappa)(\kappa-6) / 2 \kappa$ classifies the models, for instance, $\mathrm{SLE}_{6}$ and $\mathrm{SLE}_{8 / 3}$, corresponds to $c=0$, while $\mathrm{SLE}_{4}$ corresponds to $c=1$. The isolines of vorticity for the $2 \mathrm{D}$ Euler equations and the temperature isolines in the SQG model (surface of quasi-geostrophic model) belong to the class SLE, more exactly, $\mathrm{SLE}_{\kappa_{1}}$ and $\mathrm{SLE}_{\kappa_{1}}$, correspondingly, where $\kappa_{1} \approx 6$ and $\kappa_{2} \approx 4$. The energy-momentum tensor was calculated for SLE in [7].

The paper is organized as follows. Section 1 contains the results obtained (see [1-3] for more details) in a compressed form about the geometry of the correlation space $K^{3}$ and the extended group of the transformations admitted by the Lagrangian system. In Section 2, we consider the energymomentum tensor associated with the metric $d l_{D^{2}}^{2}(t)$ and show that the components $T(z)$ and $\bar{T}(\bar{z})$ of this tensor are holomorphic functions. Then, in the full analogy of CFT, we expose the asymptotic expansion both for $T(z)$ and $\bar{T}(\bar{z})$ as $z \rightarrow \infty$ and $\bar{z} \rightarrow \infty$. As a consequence of this result, we get the asymptotic expansion of the transversal correlation function for the large values of the correlation distances. Appendix to this paper is devoted to the central extension of the corresponding infinite-dimensional Lie algebra. We show that this nontrivial central extension can be determined by the same bilinear skew-symmetric form $c$ (the central charge) as for the central extension of the Witt algebra.

\section{Geometry and Group Transformations of the Correlation Space}

In this section, we demonstrate in the compressed form the results obtained in [1] about a geometric realization of the two-point velocity-correlation tensor $B_{i j}$ and present the extended symmetries of the functional of length based on the calculation of the equivalence transformations of the eikonal equation (see, for more details, [2]).

2.1. Geometric Realization of the Two-Point Velocity-Correlation Tensor. We recall only the elementary information about the structure of the two-point velocity-correlation tensor of the velocity fluctuations for homogeneous isotropic flows. The modern theory of the properties and structure of second-order (Cartesian) correlation tensors is given in [8].

The statistical description of fluid turbulence employ the Reynolds decomposition to separate the fluid velocity $\vec{u}$ at a point $\vec{x}$ into its mean and fluctuating components as $\vec{u}=\vec{u}+\vec{u}^{\prime}$. Here, (-) is the mean velocity, while $\vec{u}$ is the corresponding fluctuating quantity, usually interpreted as representing turbulence. The two-point correlation tensor is defined by the following:

$$
B_{i j}\left(x, x^{\prime}, t\right)=\overline{\left(u_{i}^{\prime}(x, t)\right)\left(u_{j}^{\prime}\left(x^{\prime}, t\right)\right)},
$$

where $t \in \mathbb{R}_{+}$and $x, x^{\prime}$ are the points of a three-dimensional space filled by turbulent fluid. Formula (2) is rewritten as follows:

$$
B_{i j}(x, \vec{r}, t)=\overline{\left(u_{i}^{\prime}(x, t)\right)\left(u_{j}^{\prime}(x+\vec{r}, t)\right)}
$$

where the vector $\vec{r}$ is determined by the pair $\left(x, x^{\prime}\right)$, where $x$ and $x^{\prime}$ are the starting point and endpoint, correspondingly, or $\vec{r}=\vec{x}^{\prime}-\vec{x}$. Therefore, we will consider an affine space $K^{3}$ with the adjoined vector space of the correlation vectors $\vec{K}^{3} \equiv\left\{\vec{r}=\left(r^{1}, r^{2}, r^{3}\right)\right\}$. The assumption of homogeneity and isotropy of turbulent flow (invariance with respect to rotation, reflection, and translation) implies that this tensor depends only on the length of the correlation vector $\vec{r}$ and time $t$; that is,

$$
\begin{aligned}
B_{i j}(x, \vec{r}, t)= & {\left[B_{L L}(|\vec{r}|, t)-B_{N N}(|\vec{r}|, t)\right] \frac{r_{i} r_{j}}{|\vec{r}|^{2}} } \\
& +B_{N N}(|\vec{r}|, t) \delta_{i j} .
\end{aligned}
$$

Moreover, for isotropic turbulence, $B_{i j}(|\vec{r}|, t)$ is a symmetric tensor and the correlations $B_{i j}$ can be expressed by using only the longitudinal correlational function $B_{L L}(|\vec{r}|, t)$ and the transversal correlation function $B_{N N}(|\vec{r}|, t)[9]$; that is, the correlation tensor $B_{i j}$ takes the diagonal form with the components $B_{11}=B_{L L}$ and $B_{22} \equiv B_{33}=B_{N N}$ in a suitable system of the coordinates of the adjoined vector space. Further instead of directly employing the correlation functions $B_{L L}$ and $B_{N N}$, we use their normalized representations $f$ and $g$ where $B_{L L}=\overline{u^{\prime 2}(t)} f(|\vec{r}|, t), B_{N N}=\overline{u^{\prime 2}(t)} g(|\vec{r}|, t)$ with

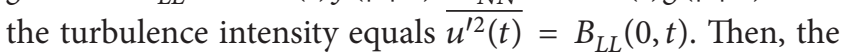


corresponding quadratic form (or linear element) takes the following form:

$$
\begin{aligned}
d l^{2}(t)= & \overline{u^{\prime 2}(t)} f(|\vec{r}|, t) d r^{1^{2}} \\
& +\overline{u^{\prime 2}(t)} g(|\vec{r}|, t)\left(d r^{2^{2}}+d r^{3^{2}}\right),
\end{aligned}
$$

where $d l^{2}(t)$ is an indefinite quadratic form in view of the properties $g(|\vec{r}|, t)$, see below. The normalized transversal correlation function $g$ satisfies the following relation (taken from the continuity) [10]:

$$
g(|\vec{r}|, t)=f(|\vec{r}|, t)+\frac{r}{2} \frac{\partial}{\partial r} f(|\vec{r}|, t) .
$$

The property that $f$ decays faster than $|\vec{r}|^{-2}$ on infinity together with (6) yields [10]

$$
\int_{0}^{\infty}|\vec{r}| g(|\vec{r}|, t) d|\vec{r}|=0
$$

Hence, $g(|\vec{r}|, t)$ is an alternative sign function. Typical forms of experimentally measured functions $f$ and $g$ are given in Figure 1. we use the data presented to determine the qualitative behaviors of $f$ and $g$, in particular, the algebraic properties of these correlation functions. Thus, we will assume that $f$ is a positive everywhere function and $g$ changes sign only in interval $\left(-\varepsilon+\left|\vec{r}^{*}\right|,\left|\vec{r}^{*}\right|+\varepsilon\right), \varepsilon>0 . g$ is a positive function on $\left[0, \pm\left|\vec{r}^{*}\right|\right)$ and therefore $g<0$ outside of $\left[-\left|\vec{r}^{*}\right|,\left|\vec{r}^{*}\right|\right]$. The change sign of $g$ means that the quadratic forms $d l^{2}(t)$ have a variable signature. The normalized longitudinal correlational function $f(|\vec{r}|, t)$ is dynamically evolved due to the von Kármán-Howarth equation [4]:

$$
\begin{aligned}
& \frac{\partial \overline{u^{\prime 2}(t)} f(|r|, t)}{\partial t} \\
& =\frac{1}{r^{4}} \frac{\partial}{\partial r} r^{4}\left({\overline{u^{\prime 2}(t)}}^{3 / 2} h(|r|, t)+2 v \frac{\partial}{\partial r} \overline{u^{\prime 2}(t)} f(|r|, t)\right) .
\end{aligned}
$$

$h$ is the normalized triple-correlation function and $\overline{u^{\prime 2}(t)}$ is the turbulence intensity (a positive everywhere function that vanishes on infinity) or the velocity scale for the turbulent kinetic energy; $\overline{u^{\prime 2}(t)}$ determines the scale for the turbulence transfer. This single equation directly follows from the Navier-Stokes equation (see, for example, [9]) and contains two unknowns, $f$ and $h$, with the turbulence intensity $\overline{u^{\prime 2}(t)}$ which cannot be defined from (8) without the use of additional hypothesis.

If we consider in the correlation space $K^{3}$ with $\vec{K}^{3}$ equipped by the standard Euclidean scalar product (i.e., in $\mathbb{R}^{3}$ ) an infinite cylindrical domain (a singled out fluid tube at some fixed time), then the metric (induced by the quadratic form $B_{i j}$ ) of the surface which bounds this domain takes the following form:

$$
\begin{aligned}
d s^{2}(t)= & \overline{u^{\prime 2}(t)} f(|\vec{r}|, t) d r^{1^{2}} \\
& +\overline{u^{\prime 2}(t)} g(|\vec{r}|, t) \rho^{2} d \phi^{2},
\end{aligned}
$$

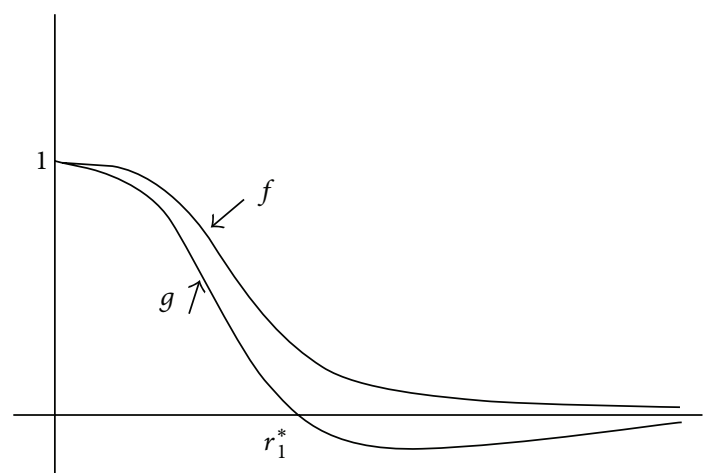

FIGURE 1: Typical forms of the normalized longitudinal and transversal correlation functions.

where $\rho$ denotes the Euclidean radius of the cross-section $\{a\} \times S^{1}(\rho)$ of the surface $\mathbb{R} \times S^{1}(\rho)$, $a \in \mathbb{R}$. We can account that $\rho=1$ and identify this manifold with $\mathbb{R} \times S^{1}(1)$. The functions $f$ and $g$ are nondimensional with $f(0, t)=$ $g(0, t)=1$ and physically $f$ is a positive function such that $f \rightarrow 0(g \rightarrow 0)$ as $|\vec{r}|$ tends to infinity. Moreover, $f$ and $g$ are bounded even functions such that $f \leq 1,|g| \leq 1$, and $f$ goes faster to zero than $|\vec{r}|^{-2}$, when $|\vec{r}|$ tends to infinity. Physically such behavior of $f$ is acceptable [10] and the map

$$
q(a, t)=\int_{0}^{a} \sqrt{f(|\vec{r}|, t)} d r^{1}, \quad a \in \mathbb{R},
$$

acts as $(-\infty, \infty) \mapsto\left[-\mathbf{L}^{*}(t), \mathbf{L}^{*}(t)\right], \mathbf{L}^{*}(t)=\mathbf{L}(t) / 2 \sqrt{\overline{u^{\prime 2}(t)}}$, where $\mathbf{L}(t)$ is determined by the following:

$$
\mathbf{L}(t)=2 \int_{0}^{\infty} \sqrt{\overline{u^{\prime 2}(t)} f(|\vec{r}|, t)} d r^{1} .
$$

Now, we rewrite the metric $d s^{2}(t)$ in the frame of the variable $q$ :

$$
\begin{gathered}
d s^{2}(t)=\overline{u^{\prime 2}(t)}\left\{d q^{2}+G(q, t) d \phi^{2}\right\}, \\
G(q, t)=g(|r|, t) .
\end{gathered}
$$

The metric (12) admits a one-parametric group of (isometric) motion $\mathbf{g}_{\tau}(\vec{p}) \equiv \mathbf{g}\left(\vec{p}, a_{1}\right), \vec{p}=(q, \phi)$ of the following form:

$$
\mathbf{g}_{\tau}:(q, \phi) \longmapsto(q, \phi+\chi \tau), \quad \chi=\text { const },
$$

with the generator

$$
X=\xi^{i} \frac{\partial}{\partial p^{i}} \equiv \chi \frac{\partial}{\partial \phi} .
$$

The scalar product of the generator $X$ equals

$$
\begin{aligned}
X^{2} & =\langle X, X\rangle=\left\langle\chi \frac{\partial}{\partial \phi}, \chi \frac{\partial}{\partial \phi}\right\rangle \equiv \chi^{2}\left\langle\frac{\partial}{\partial \phi}, \frac{\partial}{\partial \phi}\right\rangle \\
& =\overline{u^{\prime 2}(t)} \chi^{2} G(q, t)
\end{aligned}
$$

for each time $t$. 
A point $p_{0}$ is called the pole [11] of a (pseudo-) Riemannian manifold $M$ if $p_{0}$ is a fixed point of a group of diffeomorphisms $\mathbf{g}(\vec{x}, \vec{a}), \vec{a}=\left(a_{1}, \ldots, a_{r}\right)$ which acts on $M$.

We note that if $p=p_{0}\left(p_{0}\right.$ is the pole of $\left.\mathbf{g}_{\tau}\right)$, then $X^{2}\left(p_{0}\right)=$ 0 and due to (15) $p_{0}$ coincides with the roots of the equation $G(q, \cdot)=0$. Therefore, the points $q^{*} \in\left[-\mathbf{L}^{*}(t), \mathbf{L}^{*}(t)\right]$ wherein $G$ vanishes are the poles of $\mathbf{g}_{\tau}$. In view of our assumption on $g(|r|, t)$, the equation $G(q, \cdot)=0$ has only 4 roots $q_{i}^{*}, i=$ $1, \ldots, 4$ such that $\left|q_{1}^{*}\right|=q_{4}^{*}=\mathbf{L}^{*}(t)$ and $\left|q_{2}^{*}\right|=q_{3}^{*}$. Thus, the metric (12) has the different signature for $q \in I_{1}=\left(q_{2}^{*}, q_{3}^{*}\right)$, $q \in I_{2}=\left(-\mathbf{L}^{*}(t), q_{2}^{*}\right)$, and, $q \in I_{3}=\left(q_{3}^{*}, \mathbf{L}^{*}(t)\right)$, respectively, where $q_{i}^{*}$ depends on $t$. This metric determines for $q \in I_{1}$ the element of length of the surface of revolution in $\mathbb{R}^{3}$ and the radius-vector $\vec{R}=(q, \phi, \cdot)$ of this surface is given by the following:

$$
\begin{array}{r}
\vec{R}(q, \phi, \cdot)=\left(q,\left\{\overline{u^{\prime 2}(t)} G\right\}^{1 / 2}(q, \cdot) \cos \phi,\right. \\
\left.\left\{\overline{u^{\prime 2}(t)} G\right\}^{1 / 2}(q, \cdot) \sin \phi\right) .
\end{array}
$$

Therefore, the model manifold defined by (12) for $q \in I_{1}$ is a cylindrical-type surface $M_{I_{1}}^{t}=\left(q_{2}^{*}, q_{3}^{*}\right) \times S^{1}(1)$ wherein the radius of the cross-section $\{q\} \times S^{1}(1)$ equals $G^{1 / 2}(q, t)$. For the $q \in I_{i}, i=2,3$ where $G$ is negative, the positive defined metric

$$
\begin{gathered}
d s^{2}(t)=\overline{u^{\prime 2}(t)}\left\{d q^{2}-|G(q, t)| d \phi^{2}\right\}, \\
G(q, t)=g(|\vec{r}|, t),
\end{gathered}
$$

can be realized (see, for details, [1]) as a surface of revolution (for each fixed time) in the Minkowski space $\mathbb{R}_{1,2}^{3}$ with the element of length

$$
d \rho^{2}=d x_{1}^{2}-d x_{2}^{2}-d x_{3}^{2}
$$

when the form $d s^{2}(t)$ is of a fixed sign [12]. Here, the rotation presents the motion along the pseudocircle of the radius $|G(q, \cdot)|^{1 / 2}, q \in I_{i}$. Indeed, let us fix the point $p_{a}=\left(q_{a}, \phi_{a}\right)$ on the cross-section $\left\{q_{a}\right\} \times S^{1}(1)$ and consider the action of the group $\mathbf{g}_{\tau}$ on $p_{a}$; that is, the orbit $\mathbf{O}_{p_{a}}: \tau \mapsto \mathbf{g}_{\tau}\left(p_{a}\right)$. This action is a motion along $\left\{q_{a}\right\} \times S^{1}(1)$ and if $p_{a}$ does not coincide with the poles $\mathbf{g}_{\tau}$, then $\mathbf{O}_{p_{a}}$ is a not compact set [11]. In particular, $\mathbf{O}_{p_{a}} \subseteq\left\{\left(x_{1}, x_{2}\right): x_{1}^{2}-x_{2}^{2}=\overline{u^{\prime 2}(t)}\left|G\left(q_{a}, t\right)\right|\right\}$ for each fixed time $t$ which coincides with the so-called pseudocircle under the embedding $M_{I_{3}}^{t}\left(M_{I_{2}}^{t}\right)$ into the Minkowski space $\mathbb{R}_{1,2}^{3}$. Moreover, the poles are saddle points of a negative index for the orbits $\mathbf{O}_{p}, p \in M_{I_{3}}^{t}\left(M_{I_{2}}^{t}\right)$. The cross-sections $\left\{q_{a}\right\} \times S^{1}(1)$ of $M_{I_{3}}^{t}\left(M_{I_{2}}^{t}\right.$ ) for $q_{a} \in\left\{q_{3}^{*}, \mathbf{L}^{*}(t)\right\}$ (resp., $q_{a} \in\left\{-\mathbf{L}^{*}(t), q_{2}^{*}\right\}$ ) are the pseudocircles of zero radius and consist of the isotropic rays with the initial points $q_{3}^{*}$ and $\mathbf{L}^{*}(t)$ (resp., $-\mathbf{L}^{*}(t)$ and $q_{2}^{*}$ ). The action of $\mathbf{g}_{\tau}$ on the point $p$ is a motion along these piecewise linear isotropic curves when $p \in\left\{-\mathbf{L}^{*}(t), q_{2}^{*}, q_{3}^{*}, \mathbf{L}^{*}(t)\right\}$. We can identify $M_{I_{3}}^{t}\left(M_{I_{2}}^{t}\right)$ with the foliation space of orbits $M_{I_{j}}^{t}=\bigcup_{p} \mathbf{O}_{p}$ and associate the modulus of the transversal correlation function $G(q, t)$ with the length of the velocity vector $\vec{\xi}(p)$ of the orbit $\mathbf{O}_{p}$ by $|\vec{\xi}(p)|=\overline{u^{\prime 2}(t)} \chi \sqrt{|G(q, t)|}$. The length of displacement of the point $p$ (or the length of arch), with respect to the vector field generated by $\mathbf{g}_{\tau}(p)$, is determined by the formula

$$
\begin{aligned}
\lambda\left(\phi_{a}, \phi_{b}\right) & =\int_{\phi_{a}}^{\phi_{b}} \chi \sqrt{\overline{u^{\prime 2}(t)}|G(q, t)|} d \phi \\
& \equiv \chi \sqrt{\overline{u^{\prime 2}(t)}\left|G\left(q, t_{c}\right)\right|}\left(\phi_{b}-\phi_{a}\right), \quad \chi=\text { const },
\end{aligned}
$$

for each fixed time that defines the following length scale along the orbit $\mathbf{O}_{p}$ :

$$
\lambda_{\mathbf{O}_{p}}=\chi \sqrt{\overline{u^{\prime 2}(t)}|G(q, t)|} 2 \pi \phi, \quad q \in\left(q_{3}^{*}, \mathbf{L}^{*}(t)\right), \quad \chi=1 .
$$

The constant $\chi$ can be fixed by normalizing the velocity vector $\vec{\xi}(p)$.

2.2. Extended Symmetry Transformations. Let us consider again in the correlation space $K^{3}$ with $\vec{K}^{3}$ equipped by the standard Euclidean scalar product (i.e., in $\mathbb{R}^{3}$ ) an infinite cylindrical domain (a singled out fluid tube at some fixed time). Fix the cross-section $\{0\} \times D^{2}$ of this domain where $D^{2}$ is a two-dimensional disk. Then the quadratic form $\mathrm{dl}^{2}(t)$ induces on the above-mentioned cross-section the metric

$$
d l_{D^{2}}^{2}(t)=\overline{u^{\prime 2}(t)} g(|\vec{r}|, t)\left(d r^{2^{2}}+d r^{3^{2}}\right),
$$

where $|\vec{r}|=\sqrt{r^{2}+r^{3}}$. Therefore, the metric (21) is a conformal form-type metric. First, we study the case of positive values of $g(|\vec{r}|, t)$. Consider the set $\Omega\left(D^{2}, v, w\right)$ of piecewise smooth curves $\gamma: J \rightarrow D^{2}$ with fixed endpoints $\gamma(0)=v$ and $\gamma(1)=$ $w$. Let $L_{\gamma}: \Omega\left(D^{2}, v, w\right) \rightarrow \mathbb{R}$ be the paper (the simple action) for each fixed time $t$. Then the formula

$$
\begin{aligned}
& d^{t}(v, w)=\min _{\gamma \in \Omega\left(D^{2}, v, w\right)} L_{\gamma}^{t}(\gamma), \\
& L_{\gamma}^{t}=\int_{\gamma} \sqrt{\overline{u^{\prime 2}(t)} g(|\vec{r}|, t)\left(r_{\tau}^{2^{2}}+r_{\tau}^{3^{2}}\right)} d \tau,
\end{aligned}
$$

defines the function of distance $d^{t}:\left(\{0\} \times D^{2}\right) \times\left(\{0\} \times D^{2}\right) \rightarrow$ $\mathbb{R}$ on the cross-section. We can account that $\tau$ is the so-called natural parameter; that is,

$$
\overline{u^{\prime 2}(t)} g(|\vec{r}|, t)\left({r_{\tau}^{2}}^{2}+r_{\tau}^{3^{2}}\right)=1 \text { along the curve } \gamma \text {. }
$$

Therefore,

$$
L_{\gamma}^{t}=\int_{\gamma} 1 \cdot d \tau=\tau_{\gamma},
$$

where the symbol $\tau_{\gamma}$ denotes length of the curve $\gamma$. Consider the infinitesimal transformations of the variables $r^{2}$ and $r^{3}$

$$
r^{2^{*}}=r^{2}+\delta r^{2}, \quad r^{3^{*}}=r^{3}+\delta r^{3} .
$$


In order to investigate the invariance of $d^{t}$ under the action of infinitesimal transformations, it is sufficient to prove that the length of arch of extremals of the functional above is invariant. Instead of the vector $\left(r^{2}, r^{3}\right)$, we consider the unit covector $\left(\tau_{r^{2}}, \tau_{r^{3}}\right)$ defined by the formulas (for brevity we omitted the index $\gamma$ for $\tau$ ):

$$
\begin{aligned}
r_{\tau}^{2} & =\frac{\tau_{r^{2}}}{\overline{u^{\prime 2}(t)} g(|\vec{r}|, t)}, \\
r_{\tau}^{3} & =\frac{\tau_{r^{3}}}{\overline{u^{\prime 2}(t)} g(|\vec{r}|, t)} .
\end{aligned}
$$

Then (23) is transformed to

$$
\tau_{r^{2}}^{2}+\tau_{r^{3}}^{2}=\overline{u^{\prime 2}(t)} g(|\vec{r}|, t) .
$$

This equation is the eikonal-type equation. Therefore, in order to find symmetries of the functional $L_{\gamma}^{t}$, we can consider symmetries admitted by (27) that leaves $\tau$ invariant. The restatement of the variational symmetry in the terms of symmetry of partial differential equations (27) enables us to extend the class of symmetry transformations admitted by the functional $L_{\gamma}^{t}$. More exactly, we extend the variational symmetry up to the equivalence transformations. Recall that equivalence transformations for a differential equation in a given class is a change of variables which maps the equation to another equation in the same class. An equivalence transformation admitted by (27) is a point transformation given on $\left(r^{2}, r^{3}, u^{1}, u^{2}\right)$ space where $u^{1}=\tau, u^{2}=\overline{u^{\prime 2}(t)} g(|\vec{r}|, t)$. Infinitesimally, we look for an operator in the following form $[13,14]$ :

$$
\begin{aligned}
Y= & \mu\left(r^{2}, r^{3}, u^{1}, u^{2}\right) \frac{\partial}{\partial r^{2}} \\
& +v\left(r^{2}, r^{3}, u^{1}, u^{2}\right) \frac{\partial}{\partial r^{3}} \\
& +\lambda^{i}\left(r^{2}, r^{3}, u^{1}, u^{2}\right) \frac{\partial}{\partial u^{i}},
\end{aligned}
$$

where the coefficients are defined due to the following:

$$
\left.Y_{1}\right|_{(27)}=0 \text {. }
$$

Here, $Y_{1}$ denotes the first prolongation of $Y$. The infinitesimal operator $Y$ reads as follows:

$$
\begin{aligned}
Y= & \Phi\left(r^{2}, r^{3}\right) \frac{\partial}{\partial r^{2}} \\
& +\Psi\left(r^{2}, r^{3}\right) \frac{\partial}{\partial r^{2}}+\gamma\left(u^{1}\right) \frac{\partial}{\partial u^{1}} \\
& +2\left(\frac{d \gamma}{d u^{1}}-\Phi_{r^{2}}\left(r^{2}, r^{3}\right)\right) u^{2} \frac{\partial}{\partial u^{2}} .
\end{aligned}
$$

Its Lie (infinite-dimensional) subalgebra is of the following form:

$$
\begin{aligned}
X= & \Phi\left(r^{2}, r^{3}\right) \frac{\partial}{\partial r^{2}}+\Psi\left(r^{2}, r^{3}\right) \frac{\partial}{\partial r^{3}} \\
& -2 \Phi_{r^{2}}\left(r^{2}, r^{3}\right) u^{2} \frac{\partial}{\partial u^{2}}
\end{aligned}
$$

and $u^{1}(\equiv \tau)$ is a scalar invariant of $X$. Therefore, $X$ is a symmetry operator admitted by the functional $L_{\gamma}^{t}$. Here, the functions $\Phi\left(r^{2}, r^{3}\right)$ and $\Psi\left(r^{2}, r^{3}\right)$ satisfy the Cauchy-Riemann differential equations $\Phi_{r^{2}}=\Psi_{r^{3}}$ and $\Phi_{r^{3}}=-\Psi_{r^{2}}$. To get a fine structure of the equivalence transformation generated by the infinitesimal $X$, we consider the complex coordinates $z=r^{2}+i r^{3}$ and $\bar{z}=r^{2}-i r^{3}$. Then

$$
d l_{D^{2}}^{2}=\overline{u^{\prime 2}(t)} g(z \bar{z}) d z d \bar{z}
$$

or more exactly

$$
d l_{D^{2}}^{2}=\frac{\overline{u^{\prime 2}(t)} g(z \bar{z})}{2}(d z \otimes d \bar{z}+d \bar{z} \otimes d z) .
$$

The operator $X_{1}$ takes the following form:

$$
\begin{aligned}
X_{1}= & F(z) \frac{d}{d z}+\bar{F}(\bar{z}) \frac{d}{d \bar{z}} \\
& -F_{z}(z) u^{2} \frac{d}{\partial u^{2}}-\bar{F}_{\bar{z}}(\bar{z}) u^{2}(z \bar{z}) \frac{d}{\partial u^{2}} .
\end{aligned}
$$

Here, $F=\Phi+i \Psi$ and $\bar{F}=\Phi-i \Psi$, and we use that $F(z, \bar{z}) \equiv$ $F(z)$ and $\bar{F}(z, \bar{z}) \equiv \bar{F}(\bar{z})$ for the holomorphic function $F$. The tangent space is spanned by the following:

$$
\begin{aligned}
& \frac{d}{d z}=\frac{1}{2}\left(\frac{\partial}{\partial r^{2}}-i \frac{\partial}{\partial r^{3}}\right), \\
& \frac{d}{d \bar{z}}=\frac{1}{2}\left(\frac{\partial}{\partial r^{2}}+i \frac{\partial}{\partial r^{3}}\right) .
\end{aligned}
$$

For small perturbations $z \mapsto z+\epsilon(z)$ and $\bar{z} \mapsto \bar{z}+\bar{\varepsilon}(\bar{z})$, we find the representation of the operator $X_{1}$. Infinitesimal holomorphic transformations of the variables $z$ and $\bar{z}$ read as follows:

$$
\begin{gathered}
z^{*}=z+\epsilon(z)=z+F(z) \delta s \\
\bar{z}^{*}=\bar{z}+\bar{\epsilon}(\bar{z})=\bar{z}+\bar{F}(\bar{z}) \delta s
\end{gathered}
$$

which are generated by the vector field

$$
\left(F(z) \frac{d}{d z}, \bar{F}(\bar{z}) \frac{d}{d \bar{z}}\right)
$$

Using the Laurent series

$$
\begin{aligned}
& \epsilon(z)=-\sum_{n=-\infty}^{\infty} \varepsilon_{n} z^{n+1}, \\
& \bar{\epsilon}(\bar{z})=-\sum_{n=-\infty}^{\infty} \bar{\varepsilon}_{n} \bar{z}^{n+1},
\end{aligned}
$$

where $\varepsilon_{n}\left(\bar{\varepsilon}_{n}\right)$ are infinitesimal small numbers. We can look at $\varepsilon_{n} z^{n+1}, \bar{\varepsilon}_{n} \bar{z}^{n+1}$ as the harmonics of decomposition of $\epsilon(z), \bar{\epsilon}(\bar{z})$ with respect to the basis functions $\left\{z^{n+1}\right\}\left(\left\{\bar{z}^{n+1}\right\}\right)$. Each harmonics generate the transformations $z \rightarrow z^{\prime} \equiv z-$ $\varepsilon_{n} z^{n+1}, \bar{z} \rightarrow \bar{z}^{\prime} \equiv \bar{z}-\bar{\varepsilon}_{n} \bar{z}^{n+1}$ and the corresponding infinitesimal generators: $l_{n}=-z^{n+1}(d / d z), \bar{l}_{n}=-\bar{z}^{n+1}(d / d \bar{z})$, 
presents the basis of the infinite dimensional conformal Lie algebra

$$
Z=F(z) \frac{d}{d z}+\bar{F}(\bar{z}) \frac{d}{d \bar{z}} .
$$

Therefore, the basis of the operator $X$

$$
\begin{aligned}
X= & F(z) \frac{d}{d z}+\bar{F}(\bar{z}) \frac{d}{d \bar{z}} \\
& -F_{z}(z) u^{2}(z \bar{z}) \frac{d}{d u^{2}} \\
& -\bar{F}_{\bar{z}}(\bar{z}) u^{2}(z \bar{z}) \frac{d}{d u^{2}}
\end{aligned}
$$

is $k_{n} \oplus \bar{k}_{n}, n \in \mathbb{Z}$

$$
\begin{aligned}
& k_{n}=-z^{n+1} \frac{d}{d z}-(n+1) z^{n} u^{2} \frac{d}{d u^{2}} \\
& \bar{k}_{n}=-\bar{z}^{n+1} \frac{d}{d \bar{z}}-(n+1) \bar{z}^{n} u^{2} \frac{d}{d u^{2}}
\end{aligned}
$$

The factor $u^{2}$ is transformed into

$$
\begin{array}{ll}
u^{2^{*}}=\frac{u^{2}}{\left(z_{z}^{*}\right)^{2}}, & \text { for } k_{n}, \\
u^{2^{*}}=\frac{u^{2}}{\left(\bar{z}_{\bar{z}}^{*}\right)^{2}}, & \text { for } \bar{k}_{n},
\end{array}
$$

where $\left(z_{z}^{*}\right)^{2}=\left(\bar{z}_{\bar{z}}^{*}\right)^{2}$ under the change of variables $z \mapsto z^{*}$, and $\bar{z} \mapsto \bar{z}^{*}$, correspondingly.

The liner hull of the $l_{n}$ over $\mathbb{C}$ is called the Witt algebra. The Witt algebra $W$ is a dense subalgebra of the Lie algebra of holomorphic vector fields on $\mathbb{C} \backslash\{0\}$. Therefore, we can define the algebra generated by the infinitesimal operator $X$ as

$$
W_{2}=\mathbb{C}\left\{k_{n}, n \in Z\right\} \oplus \mathbb{C}\left\{\bar{k}_{n}, n \in Z\right\} .
$$

This is the linear hull of the basis elements $k_{n} \oplus \bar{k}_{n}$. Here, $k_{n}: f(z, x) \mapsto-z^{n+1} f_{z}(z, x)-(n+1) z^{n} f_{x}(z, x)$, where $z \in \mathbb{C} \backslash\{0\}, x \in \mathbb{R}_{+}$. Correspondingly for $\bar{k}_{n}$, we have $\bar{k}_{n}: f(\bar{z}, x) \mapsto-\bar{z}^{n+1} f_{\bar{z}}(\bar{z}, x)-(n+1) \bar{z}^{n} f_{x}(\bar{z}, x)$, where $\bar{z} \in \mathbb{C} \backslash\{0\}, x \in \mathbb{R}_{+} . W_{2}$ with the Lie bracket actually become Lie algebras over $\mathbb{C}$ for that we determine the Lie bracket of the $k_{n}, k_{m}$ and correspondingly of the $\bar{k}_{n}, \bar{k}_{m}$. The direct calculations demonstrate that

$$
\begin{aligned}
& {\left[k_{n}, k_{m}\right] f=k_{n} k_{m} f-k_{m} k_{n} f=(n-m) k_{n+m},} \\
& {\left[\bar{k}_{n}, \bar{k}_{m}\right] f=(n-m) \bar{k}_{n+m} f, \quad\left[k_{n}, \bar{k}_{m}\right] f=0 .}
\end{aligned}
$$

The last relation follows from the following:

$$
\frac{d}{d \bar{z}} z^{n}=0, \quad \frac{d}{d z} \bar{z}^{n}=0
$$

The commutation relations (44) and (45) will be significant for us to prove that central extension for $W_{2}$ is defined by the same skew-symmetric bilinear form as for the Witt algebra.
The case of negative values of $g(z \bar{z})$ means that $L_{\gamma}^{t}$ defines the function of imaginary distance and instead of (23) we have to consider

$$
\overline{u^{\prime 2}(t)} i^{2}|g(|\vec{r}|, t)|\left(r_{\tau}^{2^{2}}+r_{\tau}^{3^{2}}\right)=1
$$

$$
\text { along the curve } \gamma, i^{2}=-1 \text {. }
$$

The calculations of equivalence transformations of (47) are similar as for (23) where the variable $u^{2}=\overline{u^{\prime 2}(t)} g(|\vec{r}|, t)$ is replaced on $u^{2}=\overline{u^{\prime 2}(t)} i^{2}|g(|\vec{r}|, t)|$.

\section{Asymptotic Expansion of the Transversal Correlation Function}

First, using the functional of action of the trajectory $\gamma$

$$
E_{\gamma}^{t}=\int_{\gamma}\left(u^{2}\right)^{-1}\left(\tau_{r^{2}}^{2}+\tau_{r^{3}}^{2}\right) d \tau, \quad \tau_{r^{2}}^{2}+\tau_{r^{3}}^{2}=u^{2},
$$

which in the complex coordinates takes the form

$$
E_{\gamma}^{t}=4 \int_{\gamma}\left(u^{2}\right)^{-1}(z \bar{z}, t) \partial_{z} \tau(z, \bar{z}) \cdot \partial_{\bar{z}} \tau(z, \bar{z}) d \tau,
$$

we demonstrate (in the full analogy with CFT) the representation of the operators $k_{n}$ and $\bar{k}_{n}$ in terms of the components of the energy-momentum tensor obtained. Notice that this tensor has only two independent components $T(z)$ and $\bar{T}(\bar{z})$ which can be expressed by the transversal correlation function. $T(z)$ and $\bar{T}(\bar{z})$ are holomorphic functions of the variables $z$ and $\bar{z}$ whose variations under the infinitesimal transformations $z \mapsto z+\epsilon(z), \bar{z} \mapsto \bar{z}+\bar{\epsilon}(z)$ are of the form

$$
\begin{aligned}
& \delta_{\epsilon} T(z)=\epsilon(z) T^{\prime}+2 \epsilon^{\prime}(z) T(z)+(1 / 12) c \epsilon^{\prime \prime \prime}(z), \\
& \delta_{\bar{\epsilon}} \bar{T}(z)=\bar{\epsilon}(\bar{z}) \bar{T}^{\prime}+2 \overline{\epsilon^{\prime}}(\bar{z}) \bar{T}(\bar{z})+(1 / 12) \overline{c \epsilon}^{\prime \prime \prime}(\bar{z}),
\end{aligned}
$$

where $c(\bar{c})$ coincides with the central charge for the corresponding nontrivial extensions. According to [15], these formulas lead to the commutation relations for the field representations of $T(z)$ and $\bar{T}(\bar{z})$ which coincide with the commutation relations of nontrivial central extensions of the algebra $W_{2}$. Then we show how on the basis of these commutation relations to find asymptotic expansion of the transversal correlation function that present the basic interest of the theory of homogeneous isotropic turbulence.

3.1. The Energy-Momentum Tensor. Consider the functional of action of the trajectory $\gamma$

$$
E_{\gamma}^{t}=\int_{\gamma} u^{2}\left(r_{\tau}^{2^{2}}+r_{\tau}^{3^{2}}\right) d \tau
$$

and write this $E_{\gamma}^{t}$ in the form

$$
E_{\gamma}^{t}=\int_{\gamma}\left(u^{2}\right)^{-1}\left(\tau_{r^{2}}^{2}+\tau_{r^{3}}^{2}\right) d \tau, \quad \tau_{r^{2}}^{2}+\tau_{r^{3}}^{2}=u^{2} .
$$


We note that the extremals of this functional coincide with the geodesic curves and the length of the geodesic curve $\gamma$ coincides with $\tau_{\gamma}$ for the corresponding index $\gamma$. $E_{\gamma}^{t}$ admits the same infinite-dimensional Lie algebra $W_{2}$.

Recall that the classical energy-momentum tensor is defined by

$$
T_{i k}=g_{k l} f_{x^{i}}^{\alpha} \frac{\partial L}{\partial f^{\alpha} x^{l}}-g_{i k} L
$$

where $L=L\left(f^{j}, f_{x^{k}}^{i}\right)$ is a Lagrangian. For the functional (52) with the scalar field $\tau$ this tensor takes the following form:

$$
\begin{aligned}
& T_{11}=2 \tau_{r^{2}}^{2}-u^{2} \cdot 1, \\
& T_{22}=2 \tau_{r^{3}}^{2}-u^{2} \cdot 1, \\
& T_{12}=T_{21}=2 \tau_{r^{2}} \tau_{r^{3}} .
\end{aligned}
$$

$T_{i k}$ is a traceless tensor due to the equality $T_{11}+T_{22}=2\left(\tau_{r^{2}}^{2}+\right.$ $\left.\tau_{r^{3}}^{2}-u^{2}\right)=0$. Denote $\vec{\omega}=\left(r^{2}, r^{3}\right)$ and $\overrightarrow{\delta \omega}=\left(\delta r^{2}, \delta r^{3}\right)$. Then the current is defined $j_{\mu}=T_{\mu \nu} \delta \omega^{\nu}$ and has an automatically vanishing divergence $\partial \cdot j=(1 / 2) T_{\mu}^{\mu}(\partial \cdot \delta \omega)$ due to the traceless condition on $T_{\mu \nu}$.

In the complex coordinates, the functional (52) takes the following form:

$$
E_{\gamma}^{t}=4 \int_{\gamma}\left(u^{2}\right)^{-1}(z \bar{z}, t) \partial_{z} \tau(z, \bar{z}) \cdot \partial_{\bar{z}} \tau(z, \bar{z}) d \tau .
$$

Since $T_{i k}$ is traceless, we have $T_{z \bar{z}}=T_{\bar{z} z}=0$; these imply $\partial_{\bar{z}} T_{z z}=\partial_{z} T_{\overline{z z}}=0$ and there are only two nonvanishing components of the energy-momentum tensor $T(z)=T_{z z}(z)=1 / 4\left(T_{11}-2 i T_{12}-T_{22}\right)$ and $\bar{T}(\bar{z})=$ $T_{\overline{z z}}(\bar{z})=1 / 4\left(T_{11}+2 i T_{12}-T_{22}\right)$. Moreover $T_{z z}=\left(\partial_{z} \tau\right)^{2}$ and $T_{\overline{z z}}=\left(\partial_{\bar{z}} \tau\right)^{2}$. In the line of Conformal Field Theory, we can consider the following constructions: since $T(z)$ and $\bar{T}(\bar{z})$ are holomorphic functions, then due to Laurent expansions

$$
T(z)=\sum_{n} L_{n} z^{-n-2}, \quad \bar{T}(\bar{z})=\sum_{n} \bar{L}_{n} \bar{z}^{-n-2},
$$

where the exponent $-n-2$ is chosen so that for the scale transformation $z \mapsto \lambda^{-1} z$ under which $T(z) \mapsto \lambda^{2} T\left(\lambda^{-1} z\right)$ we have $L_{-n} \mapsto \lambda^{n} L_{-n}$ and $\bar{L}_{-n} \mapsto \bar{\lambda}^{n} \bar{L}_{-n}$. Therefore, the expression (56) is finally inverted by the following relations:

$$
L_{n}=\frac{1}{2 \pi i} \oint T(z) z^{n+1} d z, \quad \bar{L}_{n}=\frac{1}{2 \pi i} \oint \bar{T}(\bar{z}) \bar{z}^{n+1} d \bar{z} .
$$

Recall that $L_{n}$ are defined on $\mathbb{C} \backslash\{0\}$ :

$$
T_{\epsilon}=\oint \epsilon(z) T(z) d z, \quad \bar{T}_{\bar{\epsilon}}=\oint \bar{\epsilon}(\bar{z}) \bar{T}(\bar{z}) d z
$$

Their variations read as follows:

$$
\begin{aligned}
& \delta_{\epsilon} T(z)=\epsilon(z) T^{\prime}+2 \epsilon^{\prime}(z) T(z)+\left(\frac{1}{12}\right) c \epsilon^{\prime \prime \prime}(z) \\
& \delta_{\bar{\epsilon}} \bar{T}(z)=\bar{\epsilon}(\bar{z}) \bar{T}^{\prime}+2 \overline{\epsilon^{\prime}}(\bar{z}) \bar{T}(\bar{z})+\left(\frac{1}{12}\right) \overline{c \epsilon}^{\prime \prime \prime}(\bar{z}) .
\end{aligned}
$$

It leads to (see [15])

$$
\begin{gathered}
{\left[T_{\epsilon}, T(z)\right]=\epsilon(z) T^{\prime}(z)+2 \epsilon(z) T(z)+\frac{c}{12} \epsilon^{\prime \prime \prime}(z),} \\
{\left[\bar{T}_{\bar{\epsilon}}, \bar{T}(\bar{z})\right]=\bar{\epsilon}(\bar{z}) \bar{T}^{\prime}(\bar{z})+2 \bar{\epsilon}(\bar{z}) \bar{T}(\bar{z})+\frac{c}{12} \bar{\epsilon}^{\prime \prime \prime}(\bar{z}) .}
\end{gathered}
$$

Then from (60) we get as in [15] that $L_{n}$ and $\bar{L}_{n}$ satisfy the commutation relations

$$
\begin{aligned}
{\left[L_{n}, L_{m}\right]=} & (n-m) L_{n+m}+\frac{c}{12} \delta_{n+m} n\left(n^{2}-1\right), \\
{\left[\bar{L}_{n}, \bar{L}_{m}\right]=} & (n-m) \bar{L}_{n+m} \\
& +\frac{\bar{c}}{12} \delta_{n+m} n\left(n^{2}-1\right), \quad\left[L_{n}, \bar{L}_{m}\right]=0 .
\end{aligned}
$$

Lemma 1. Let $T(z)=\sum_{n} L_{n} z^{-n-2}$ and $T(w)=\sum_{n} L_{n} w^{-n-2}$ be the fields with $\left\{L_{n}\right\}$ satisfying the commutation relationship (61). Then for large $z$ and $w$, it holds that

$$
T(z) T(w) \sim \frac{c}{2(z-w)^{4}}+\frac{2 T(w)}{(z-w)^{2}}+\frac{\partial_{w} T(w)}{z-w} .
$$

The left-hand side of (61) reads as follows:

$$
\begin{aligned}
{\left[L_{n}, L_{m}\right]=} & \left(\oint_{|z|>|w|} \frac{d z}{2 \pi i} \oint \frac{d w}{2 \pi i}-\oint \frac{d w}{2 \pi i} \oint_{|z|<|w|} \frac{d z}{2 \pi i}\right) \\
& \times z^{n+1} T(Z) w^{n+1} T(w) .
\end{aligned}
$$

The proof of this lemma is based on the direct calculations. The first term of the right-hand side of (61) equals

$$
(n-m) L_{n+m}=(n-m) \oint \frac{d w}{2 \pi i}\left(w^{n+m+1} T(w)\right) .
$$

This integral can be rewritten as follows

$$
\oint \frac{d w}{2 \pi i}\left(2(n+1) w^{n} w^{m+1} T(w)+w^{n+1} w^{m+1} \partial_{w} T(w)\right) .
$$

Indeed, integrating the last term of this integral by parts and combining with the first term we get the right-hand part of (65). The second term of (61), that is, $(c / 12) \delta_{n+m} n\left(n^{2}-1\right)$, can be presented as the following integral:

$$
\oint \frac{d w}{2 \pi i}\left(\frac{c}{12} n\left(n^{2}-1\right) w^{n-2} w^{m}\right) .
$$

Further transformations are based on using the well-known formula from the Complex Analysis

$$
\partial_{\zeta}^{n} f(\zeta)=n ! \oint \frac{d w}{2 \pi i} \frac{f(\zeta)}{(\zeta-z)^{n}}
$$

Since $\left.\partial_{z}^{3} z^{n+1}\right|_{z=w}=n\left(n^{2}-1\right) w^{n-2}$, then applying (68), we get that (67) equals

$$
\oint \frac{d z}{2 \pi i} \oint \frac{d w}{2 \pi i} z^{n+1} w^{m+1} \frac{c}{2(z-w)^{4}} .
$$


Applying the same procedure to the first and the second terms of (66), we can see that this integral equals

$$
\oint \frac{d z}{2 \pi i} \oint \frac{d w}{2 \pi i} z^{n+1} w^{m+1}\left(\frac{2 T(w)}{(z-w)^{2}}+\frac{\partial T(w)}{z-w}\right) .
$$

Combining (69) and (70), we found that the integral

$$
\oint \frac{d z}{2 \pi i} \oint \frac{d w}{2 \pi i} z^{n+1} w^{m+1}\left(\frac{c}{2(z-w)^{4}}+\frac{2 T(w)}{(z-w)^{2}}+\frac{\partial T(w)}{z-w}\right)
$$

equals the right-hand side of (64). For the completeness, we need to add at the integral (71) a regular function $R(z, w)$ on the variable $z-w$ since this function is free of poles at $z=w$; hence, $R(z, w)$ does not contribute to the integral. Therefore, comparing (71) with (64), we derive that

$$
T(z) T(w)=\frac{c}{2(z-w)^{4}}+\frac{2 T(w)}{(z-w)^{2}}+\frac{\partial_{w} T(w)}{z-w}+R(z, w) .
$$

This relationship is called the operator product expansion in CFT. The same assertion holds for the $\bar{T}(\bar{z})$. Notice that the quantities $c$ and $\bar{c}$ coincide.

Corollary 2. Consider the following:

$$
T(z) T(0) \sim \frac{c}{2 z^{4}}, \quad \bar{T}(\bar{z}) \bar{T}(0) \sim \frac{c}{2 \bar{z}^{4}} \quad \text { as } z, \bar{z} \longrightarrow \infty .
$$

One applies the results obtained to find asymptotic behavior of the transversal correlation function $B_{N N}=\overline{u^{\prime 2}(t)} g(z \bar{z})$ as the length of the correlation vector tends to infinity. For this, one uses the following relationship:

$$
4 \partial_{z} \tau \cdot \partial_{\bar{z}} \tau=u^{2}
$$

and the equalities $T(z)=T_{z z}=\left(\partial_{z} \tau\right)^{2}$ and $\bar{T}(\bar{z})=T_{\overline{z z}}=$ $\left(\partial_{\bar{z}} \tau\right)^{2}$. Rewrite (74) in the form $\left(\partial_{z} \tau\right)^{2} \cdot\left(\partial_{\bar{z}} \tau\right)^{2}=(1 / 16)\left(u^{2}\right)^{2}$ and use the formulas (73). Then substituting instead of $\left(u^{2}\right)^{2}$ the quantity ${\overline{u^{\prime 2}(t)}}^{2} g^{2}(|\vec{r}|, t)$ in (73), one gets the asymptotic expansion for $B_{N N}^{2}(|\vec{r}|, t)$ in the follwing form:

$$
\begin{aligned}
& {\overline{u^{\prime 2}(t)}}^{2} g^{2}(|\vec{r}|, t) \sim \frac{4 c^{2}}{|\vec{r}|^{8} T(0) \bar{T}(0)}, \\
& z \bar{z}=|\vec{r}|^{2} \quad \text { as }|\vec{r}| \longrightarrow \infty .
\end{aligned}
$$

Since $T(0) \bar{T}(0)=(1 / 16){\overline{u^{\prime 2}(t)}}^{2}$, one finally obtains from (75) that

$$
B_{N N}(|\vec{r}|, t) \sim-\frac{8 c}{{\overline{u^{\prime 2}(t)}}^{2}|\vec{r}|^{4}} .
$$

The sign "-" appears in view of the negative values of $B_{N N}(|\vec{r}|, t)$ as $|\vec{r}| \rightarrow \infty$.

\section{Concluding Remarks}

The behavior of the correlation functions $B_{L L}$ and $B_{N N}$ presents significant interests for the theory of turbulence since this leads to various types of the so-called integral invariants. The Loitsyansky and Birkhoff integrals are the most famous integral invariants. In this paper, we established the asymptotic behavior of the transversal correlation function $B_{N N}(|\vec{r}|, t)$ as $|\vec{r}| \rightarrow \infty$ for the geometry of the correlation space $K^{3}$ determined by the two-point velocitycorrelation tensor in the case of homogeneous isotropic turbulence. The question about the asymptotic expansion of $B_{N N}(|\vec{r}|, t)$ for large values of the correlation distances in the physical space $\mathbb{R}^{3}$ with the standard Euclidian metric is still open. Nevertheless, the formula (76) can be applied for studying this problem if we construct the isometric embedding of the couple $\left(K^{3}, d l^{2}(t)\right)$ into the physical space that presents another topic of investigations.

\section{Appendix}

In this section, we show that nontrivial central extension of the Lie algebra $W_{2}$ exists.

In order to avoid a redundant complexity of the exposition of material, we recall basic definitions and present only elementary results on the algebraic constructions concerning central extensions of the Lie algebras. We begin several definitions.

A Lie algebra $\mathbf{a}$ is called abelian if Lie bracket of $\mathbf{a}$ is trivial; that is, $[X, Y]=0$ for all $X, Y \in \mathbf{a}$.

Let $\mathbf{a}$ be an abelian Lie algebra over $\mathbb{C}$ and $\mathbf{g}$ a Lie algebra over $\mathbb{C}$. An exact sequence of algebra homomorphisms

$$
0 \longrightarrow \mathbf{a} \longrightarrow \mathbf{b} \longrightarrow \mathbf{g} \longrightarrow 0
$$

is called a central extension of $\mathbf{g}$ by $\mathbf{a}$, if $[\mathbf{a}, \mathbf{g}]=0$; that is, $[X, Y]=0$ for all $X \in \mathbf{a}$ and $Y \in \mathbf{b}$.

Here, we identify a with the corresponding subalgebra of b. For such a central extension, the abelian Lie algebra a is realized as an ideal in $\mathbf{b}$ and the homomorphism $\pi: \mathbf{b} \mapsto \mathbf{g}$ serves to identify $\mathbf{g}$ with $\mathbf{b} / \mathbf{a}$.

For every central extension of the Lie algebras

$$
0 \longrightarrow \mathbf{a} \longrightarrow \mathbf{b} \longrightarrow \mathbf{g} \longrightarrow 0,
$$

there is a linear map $\beta: \mathbf{g} \mapsto \mathbf{b}$ with $\pi \cdot \beta=i d_{\mathbf{b}}$. Here, $\beta$ is in general not a Lie algebra homomorphism. Consider

$$
\Theta(X, Y)=[\beta(X), \beta(Y)]-\beta([X, Y]) \quad \text { for } X, Y \in \mathbf{g} .
$$

Then the map $\Theta: \mathbf{g} \times \mathbf{g} \mapsto \mathbf{a}$ (depending on $\beta$ ) always has the following properties:

(a) $\Theta: \mathbf{g} \times \mathbf{g} \mapsto \mathbf{a}$ is a skew-symmetric bilinear form;

(b) $\Theta(X,[Y, Z])+\Theta(Y,[Z, X])+\Theta(Z,[X, Y])=0$.

Moreover, $\mathbf{b}$ is isometric to $\mathbf{g} \oplus \mathbf{a}$ as vector spaces by the linear isomorphism

$$
\psi: \mathbf{g} \times \mathbf{a} \longmapsto \mathbf{b}, \quad X \oplus Y=(X, Y) \longmapsto \beta(X)+Y,
$$


Thus, with the Lie bracket on $\mathbf{g} \oplus \mathbf{a}$ given by

$$
\left[X \oplus Z, Y \oplus Z^{\prime}\right]_{\mathbf{b}}=[X, Y]_{\mathbf{g}}+\Theta(X, Y)
$$

for $X, Y \in \mathbf{g}$ and $Z, Z^{\prime} \in \mathbf{a}$, the map $\psi$ is a Lie algebra isomorphism.

The Lie bracket on $\mathbf{b}$ can also be written as

$$
\left[\beta(X)+Z, \beta(Y)+Z^{\prime}\right]=\beta([X, Y])+\Theta(X, Y) .
$$

A map $\Theta: \mathbf{b} \times \mathbf{b} \mapsto \mathbf{a}$ with the properties (a) and (b) is called a 2-cocycle on $\mathbf{g}$. hold.

With the definitions introduced, the following results

Lemma A.1. Every central extension $\mathbf{b}$ of $\mathbf{g}$ by a comes from a cocycle $\Theta: \mathbf{b} \times \mathbf{b} \mapsto \mathbf{a}$. Every cocycle $\Theta: \mathbf{b} \times \mathbf{b} \mapsto \mathbf{a}$ generates a central extension $\mathbf{b}$ of $\mathbf{g}$ by $\mathbf{a}$.

The first result follows from the comments above. Let $\mathbf{b}$ be the vector space $\mathbf{b}=\mathbf{g} \oplus \mathbf{a}$. The bracket

$$
\left[X \oplus Z, Y \oplus Z^{\prime}\right]_{\mathbf{b}}=[X, Y]_{\mathbf{g}} \oplus \Theta(X, Y)
$$

for $X, Y \in \mathbf{g}$ and $Z, Z^{\prime} \in \mathbf{a}$ is a Lie bracket if and only if $\Theta$ is a cocycle. Hence, $\mathbf{b}$ with such Lie bracket defines a central extension of $\mathbf{g}$ by $\mathbf{a}$ that proves the second assertion of this lemma.

Define the following sets:

$$
\begin{aligned}
\operatorname{Alt}^{2}(\mathbf{g}, \mathbf{a}) & =\{\Theta: \mathbf{b} \times \mathbf{b} \longmapsto \mathbf{a} \mid \Theta \text { satisfies condition } \mathbf{a})\} \\
\mathbf{Z}^{2}(\mathbf{g}, \mathbf{a}) & \left.=\left\{\Theta \in \mathbf{A l t}^{2}(\mathbf{g}, \mathbf{a}) \mid \Theta \text { satisfies condition } \mathbf{b}\right)\right\}
\end{aligned}
$$

$\mathbf{Z}^{2}$ is a linear subspace of $\mathbf{A l t} \mathbf{t}^{2}$. The above vector spaces are abelian groups, and $\mathbf{Z}^{2}$ is the space of 2 cocycles.

Let us consider the Lie algebra $W_{2}$ and denote the corresponding components $\mathbb{C}\left\{k_{n}, n \in Z\right\} \oplus \mathbb{C}\left\{\bar{k}_{n}, n \in Z\right\}$ for these algebras by $W_{2}^{a}, W_{2}^{b}$. In order to find central extension of $W_{2}$, it is sufficient to construct a central extension for each component.

Lemma A.2. The central extension $E W_{2}^{a}$ of $W_{2}^{a}$ by $Z$ exists; that is,

$$
\begin{gathered}
E W_{2}^{a}=W_{2}^{a} \oplus \mathbb{C} Z \quad \text { as a complex vector space, } \\
{\left[k_{n}, k_{m}\right]=(n-m)+\frac{c}{12} \delta_{n+m} n\left(n^{2}-1\right), \quad c \in \mathbb{C},} \\
{\left[k_{n}, Z\right]=0 \quad \text { for } n, m \in Z .}
\end{gathered}
$$

This central extension is defined by the cocycle $\Theta \in \mathbf{Z}^{2}\left(\mathbf{W}_{2}^{\mathbf{a}}\right)$, $\Theta=c \omega$, where $\omega=(1 / 12) \delta_{n+m} n\left(n^{2}-1\right)$ which coincides with the cocycle of the Witt algebra $W$.

The proof of this lemma completely repeats one as we came to the Virasoro algebra Vir which is a proper central extension of the Witt algebra $W$ (see, for more details, [16]). For the convince, we shortly present this proof again.
First, we establish that $\Theta \in \mathbf{Z}^{2}\left(\mathbf{W}_{2}^{\mathbf{a}}\right)$; that is, we have to check that

$$
\Theta\left(k_{s},\left[k_{m}, k_{n}\right]\right)+\Theta\left(k_{m},\left[k_{n}, k_{s}\right]\right)+\Theta\left(k_{n},\left[k_{s}, k_{m}\right]\right)=0
$$

for $s, m, n \in Z$. Using the commutation relations (44), the direct calculations show that

$$
\begin{aligned}
12\left(\Theta\left(k_{s},\left[k_{m}, k_{n}\right]\right)+\Theta\left(k_{m},\left[k_{n}, k_{s}\right]\right)+\Theta\left(k_{n},\left[k_{s}, k_{m}\right]\right)\right) \\
=c \delta_{s+m+n}\left((m-n) s\left(s^{2}-1\right)+(n-s) m\left(m^{2}-1\right)\right. \\
\left.\quad+(s-m) n\left(n^{2}-1\right)\right) \\
=-c((m-n)(m+n) \\
\quad \times\left((m+n)^{2}-1\right)+(2 n+m) m\left(m^{2}-1\right) \\
\left.\quad-(2 m+n) n\left(n^{2}-1\right)\right)=0 .
\end{aligned}
$$

That proves the inclusion $\Theta \in \mathbf{Z}^{2}\left(\mathbf{W}_{2}^{\mathbf{a}}\right)$. Further, we have

$$
\begin{aligned}
& 0=\Theta\left(k_{s},\left[k_{m}, k_{n}\right]\right)+\Theta\left(k_{m},\left[k_{n}, k_{s}\right]\right)+\Theta\left(k_{n},\left[k_{s}, k_{m}\right]\right) \\
& =c\left((m-n) \Theta\left(k_{s}, k_{m+n}\right)+(n-s) \Theta\left(k_{m}, k_{n+s}\right)\right. \\
& \left.\quad+(k-m) \Theta\left(k_{n}, k_{s+m}\right)\right) .
\end{aligned}
$$

For $s=0$ we get

$$
(m-n) \Theta\left(k_{0}, k_{m+n}\right)+n \Theta\left(k_{m}, k_{n}\right)-m \Theta\left(k_{n}, k_{s+m}\right)=0 .
$$

Hence,

$$
\Theta\left(k_{n}, k_{m}\right)=\frac{m-n}{m+n} \Theta\left(k_{0}, k_{m+n}\right),
$$

for $m, n \in Z, m \neq-n$.

Define a homomorphism $\mu \in \operatorname{Hom}_{\mathbb{C}}\left(W_{2}^{a}, \mathbb{C}\right)$ by

$$
\begin{gathered}
\mu\left(k_{n}\right)=\frac{1}{n} \Theta\left(k_{0}, k_{n}\right) \quad \text { for } n \in Z \backslash\{0\}, \\
\mu\left(k_{0}\right)=-\frac{1}{2} \Theta\left(k_{1}, k_{-1}\right),
\end{gathered}
$$

and let $\Theta^{\prime}=\Theta+\widetilde{\mu}, \tilde{\mu}(X, Y)=\mu([X, Y])$. Then, $\Theta^{\prime}\left(k_{n}, k_{m}\right)=0$ for $m, n \in Z, m \neq-n$, since

$$
\begin{aligned}
\Theta^{\prime}\left(k_{n}, k_{m}\right) & =\Theta\left(k_{n}, k_{m}\right)+\mu\left(\left[k_{n}, k_{m}\right]\right) \\
& =\frac{m-n}{m+n} \Theta\left(k_{0}, k_{n+m}\right)+\mu\left((n-m)\left[k_{n+m}\right]\right) \\
& =\frac{m-n}{m+n} \Theta\left(k_{0}, k_{n+m}\right)+\frac{n-m}{m+n} \Theta\left(k_{0}, k_{n+m}\right)=0 .
\end{aligned}
$$

Thus, there is a map $h: Z \mapsto \mathbb{C}$ such that

$$
\Theta^{\prime}\left(k_{n}, k_{m}\right)=\delta_{n+m} h(n) \quad \text { for } n, m \in Z .
$$


Since $\Theta^{\prime}$ is skew-symmetric, it follows that

$$
h(0)=0, \quad h(-s)=-h(s) \quad \forall n, m \in Z .
$$

By definition of $\mu$, we have

$$
\begin{aligned}
h(1) & =\Theta^{\prime}\left(k_{1}, k_{-1}\right) \\
& =\Theta\left(k_{1}, k_{-1}\right)+\mu\left(\left[k_{1}, k_{-1}\right]\right) \\
& =\Theta\left(k_{1}, k_{-1}\right)-\Theta\left(k_{1}, k_{-1}\right) \\
& =0 .
\end{aligned}
$$

It remains to be shown that there is a $c \in \mathbb{C}$ with $\Theta^{\prime}=c \omega$; that is,

$$
h(n)=\frac{c}{12} n\left(n^{2}-1\right) \quad \text { for } n \in \mathbb{N} .
$$

Since $\Theta^{\prime} \in \mathbf{Z}^{2}\left(\mathbf{W}_{\mathbf{2}, \mathbf{0}}^{\mathbf{2}, \mathbf{a}}\right)$, we have for $s, m, n \in \mathbb{N}$ the following:

$$
\begin{aligned}
0= & \Theta^{\prime}\left(k_{s},\left[k_{m}, k_{n}\right]\right)+\Theta\left(k_{m},\left[k_{n}, k_{s}\right]\right)+\Theta\left(k_{n},\left[k_{s}, k_{m}\right]\right) \\
= & c\left((m-n) \Theta^{\prime}\left(k_{s}, k_{m+n}\right)+(n-s) \Theta^{\prime}\left(k_{m}, k_{n+s}\right)\right. \\
& \left.+(k-m) \Theta^{\prime}\left(k_{n}, k_{s+m}\right)\right) .
\end{aligned}
$$

For $k+m+n=0$, we obtain

$$
\begin{aligned}
0= & (m-n) h(s)+(n-s) h(m) \\
& +(s-m) h(n) \\
= & -(m-n) h(m+)(2 n+m) h(m) \\
& -(2 m+n) h(n) .
\end{aligned}
$$

The substitution $n=1$ yields the following:

$$
-(m-1) h(m+1)+(2+m) h(m)-(2 m+1) h(1)=0,
$$

for $m \in \mathbb{N}$. Combined with $h(1)$, this implies the recursion formula

$$
h(m+1)=\frac{m+2}{m-1} h(m) \quad \text { for } m \in \mathbb{N} .
$$

Consequently, the map $h$ is completely determined by $h(2) \epsilon$ $\mathbb{C}$. We can show by induction $n \in \mathbb{N}$ that for $c \in 2 h(2)$ the relation (A.20) holds. Cases $n=1$ and $n=2$ are obvious. So let $m=m \in \mathbb{N}, n>1$, and $h(m)=(c / 12) m\left(m^{2}-1\right)$. Then,

$$
\begin{aligned}
h(m+1) & =\frac{m+2}{m-1} h(m) \\
& =\frac{m+2}{m-1} \frac{c}{12} m\left(m^{2}-1\right) \\
& =\frac{c}{12} m((m+1)(m+2)) \\
& =\frac{c}{12}(m+1)\left((m+1)^{2}-1\right) .
\end{aligned}
$$

That completes the proof of Lemma A.2.
Corollary A.3. The Lie algebra $E W_{2}^{a}$ is homeomorphic to the Virasoro algebra $W$.

Lemma A.2 and Corollary A.3 still hold if we consider $W_{2}^{b}$ instead of $W_{2}^{a}$ and change $W$ by $\bar{W}$ for the corresponding basis elements $\bar{l}_{n}=-\bar{z}^{n+1} d / d \bar{z}$ of $\bar{W}$ (a copy of $W$ ).

\section{Acknowledgments}

This work was supported by FAPESP (Grant no. 11/50984-1), RFBR (Grant no. 12-01-00648), RFBR (Grant no. 11-01-12075OFIM-2011), and partially by Grant no. 12.740.11.1430 of the Government of Russian Federation.

\section{References}

[1] V. N. Grebenev and M. Oberlack, "Geometric realization of the two-point velocity correlation tensor for isotropic turbulence," Journal of Nonlinear Mathematical Physics, vol. 18, no. 1, pp. 109120, 2011.

[2] V. N. Grebenev, M. Oberlack, and A. N. Grishkov, "Infinite dimensional Lie algebra associated with conformal transformations of the two-point velocity correlation tensor from isotropic turbulence," Zeitschrift für angewandte Mathematik und Physik.

[3] V. N. Grebenev and M. Oberlack, "A geometry of the correlation space and a nonlocal degenerate parabolic equation from isotropic turbulence," Zeitschrift für Angewandte Mathematik und Mechanik, vol. 92, no. 3, pp. 179-195, 2012.

[4] Th. von Kármán and L. Howarth, "On the statistical theory of isotropic turbulence," Proceedings of the Royal Society A, vol. 164, pp. 192-215, 1938.

[5] G. Falkovich, "Conformal invariance in hydrodynamic turbulence," Russian Mathematical Surveys, vol. 62, no. 3, pp. 497-510, 2007.

[6] D. Bernard, G. Bofffeta, A. Celani, and G. Falkovich, "Conformal invariance in two-dimensional turbulence," Nature Physics, vol. 2, pp. 124-128, 2006.

[7] J. Cardy, "The power of two dimensions," Nature Physics, vol. 2, pp. 67-68, 2006.

[8] S. Oughton, K.-H. Rädler, and W. H. Matthaeus, "General second-rank correlation tensors for homogeneous magnetohydrodynamic turbulence," Physical Review E, vol. 56, no. 3, pp. 2875-2888, 1997.

[9] A. S. Monin and A. M. Yaglom, Statistical Hydromechanics, Gidrometeoizdat, St. Petersburg, Russia, 1994.

[10] J. C. Rotta, Turbulente Strömungen, Teubner, Stuttgar, Russia, 1972.

[11] N. R. Kamyshanskij and A. S. Solodovnikov, "Semireducible analytic spaces "in the large",' Russian Mathematical Surveys, vol. 35, no. 5, pp. 1-56, 1980.

[12] L. P. Eisenhart, Riemannian Geometry, Princeton University Press, Princeton, NJ, USA, 1926.

[13] A. G. Megrabov, "Group spliting and Lax representation," Doklady Mathematics, vol. 67, no. 3, pp. 335-349, 2003.

[14] S. V. Meleshko, "Homogeneous autonomous systems with three independent variables," Journal of Applied Mathematics and Mechanics, vol. 58, no. 5, pp. 857-863, 1994.

[15] A. A. Belavin, A. M. Polyakov, and A. B. Zamolodchikov, "Infinite conformal symmetry in two-dimensional quantum field theory," Nuclear Physics B, vol. 241, no. 2, pp. 333-380, 1984. 
[16] M. Schottenloher, A Mathematical Introduction to Conformal Field Theory, vol. 759 of Lecture Notes in Physics, Springer, Berlin, Germany, 2nd edition, 2008. 


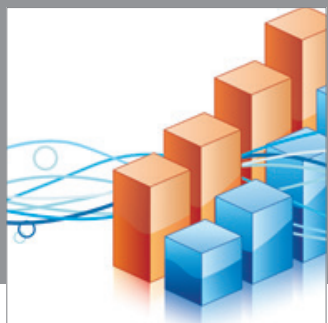

Advances in

Operations Research

mansans

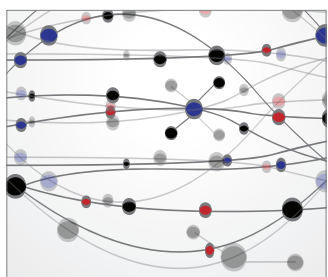

The Scientific World Journal
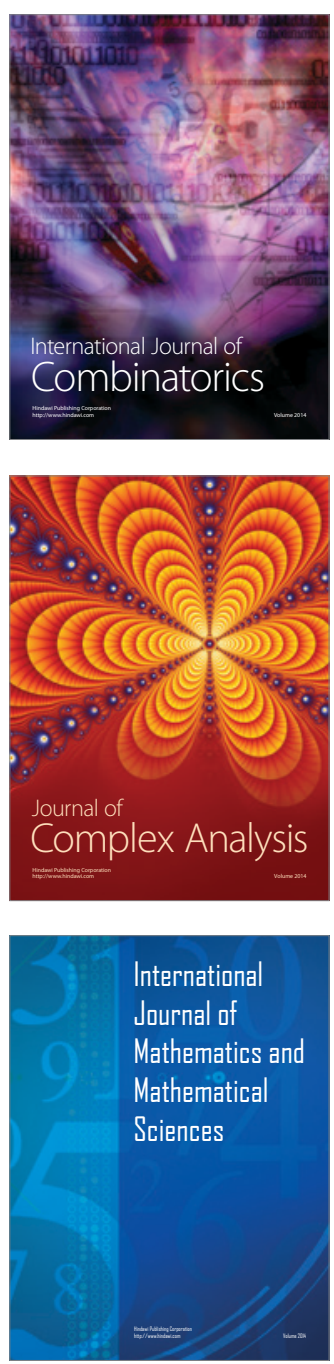
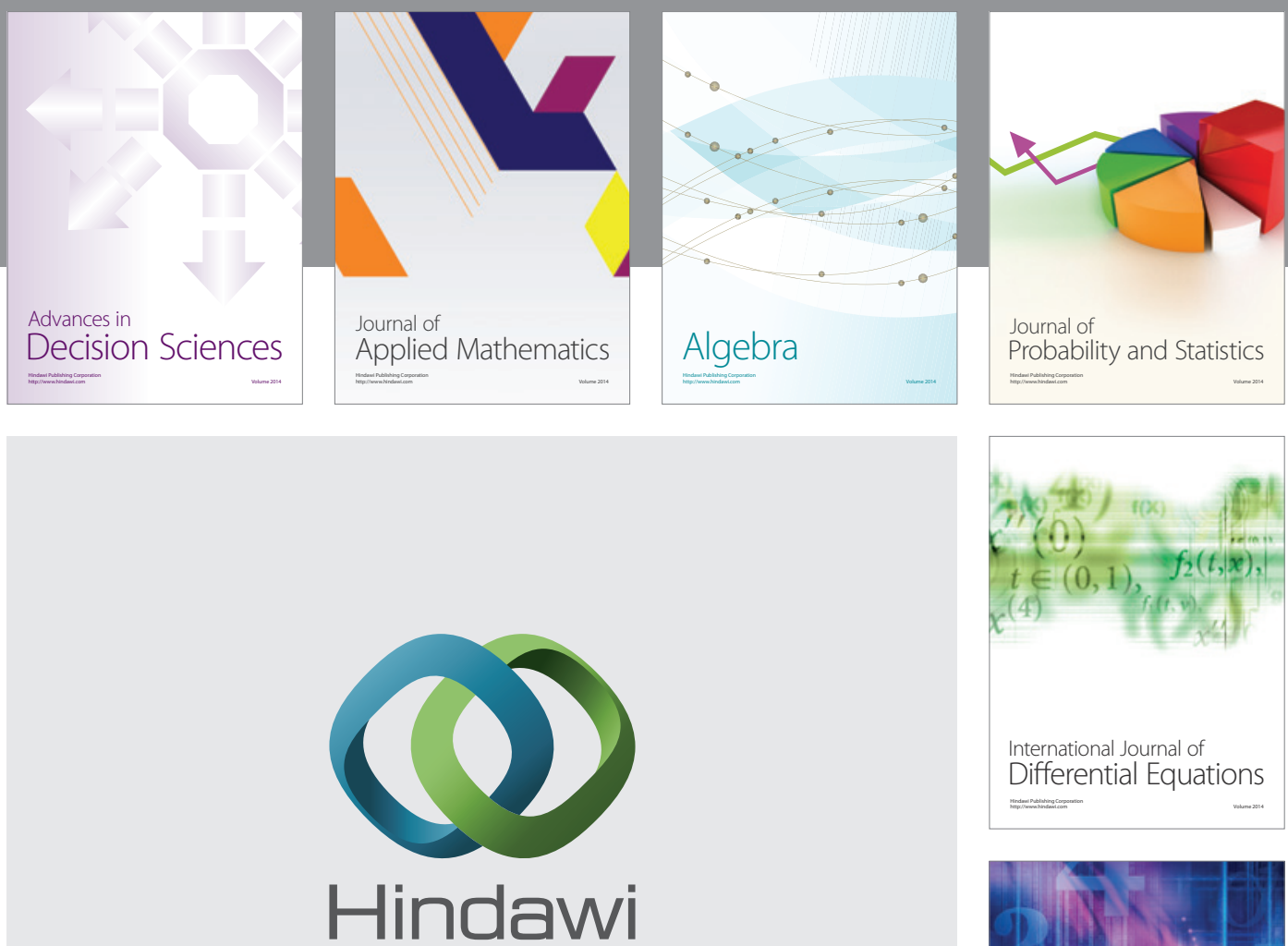

Submit your manuscripts at http://www.hindawi.com
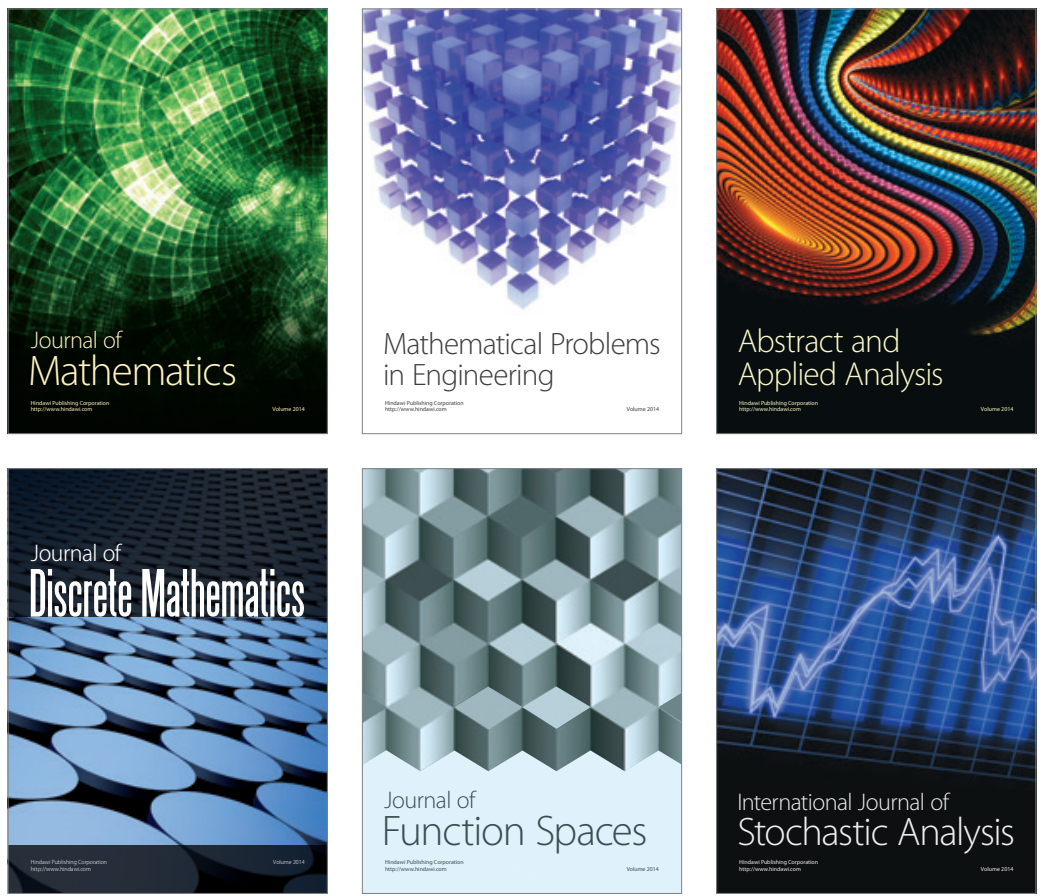

Journal of

Function Spaces

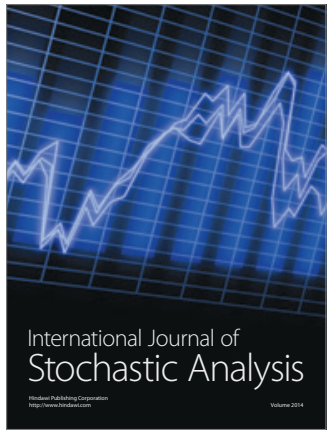

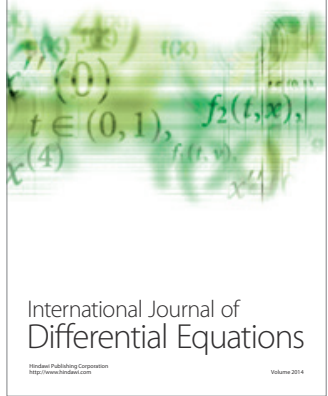
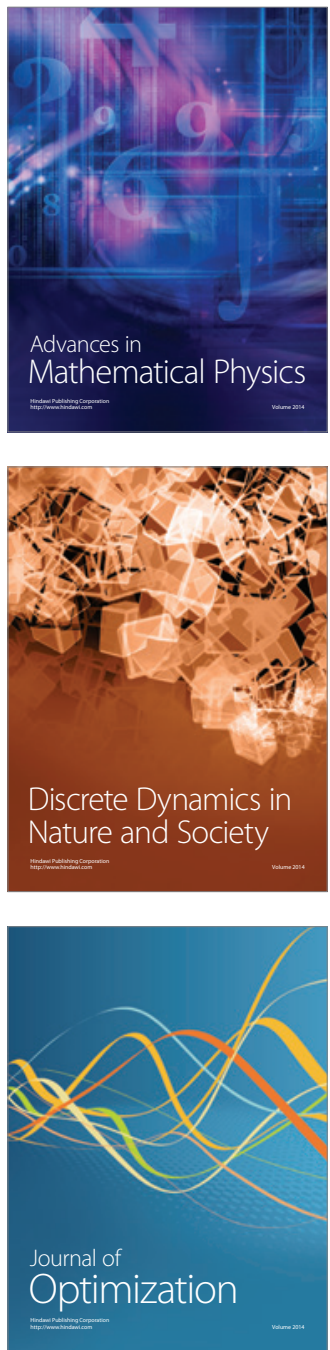